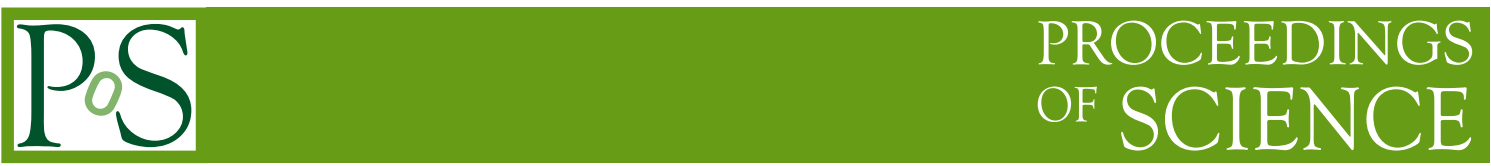

\title{
Charmed Bottom Baryon Spectroscopy
}

\section{Zachary S. Brown}

The College of William \& Mary

E-mail: zsbrown@email.wm.edu

\section{William Detmold}

Massachusetts Institute of Technology

E-mail: wdetmoldemit.edu

\section{Stefan Meinel}

Massachusetts Institute of Technology

E-mail: smeinel@mit.edu

\section{Kostas Orginos}

The College of William \& Mary

E-mail: kostas@wm. edu

\begin{abstract}
The spectrum of doubly and triply heavy baryons remains experimentally unexplored to a large extent. Although the detection of such heavy particle states may lie beyond the reach of experiments for some time, it is interesting compute this spectrum from QCD and compare results between lattice calculations and continuum theoretical models. Several lattice calculations exist for both doubly and triply charmed as well as doubly and triply bottom baryons. Here, we present preliminary results from the first lattice calculation of doubly and triply heavy baryons including both charm and bottom quarks. We use domain wall fermions for $2+1$ flavors (up down and strange) of sea and valence quarks, a relativistic heavy quark action for the charm quarks, and non-relativistic QCD for the heavier bottom quarks. We present preliminary results for the ground state spectrum.
\end{abstract}

31st International Symposium on Lattice Field Theory - LATTICE 2013

July 29 - August 3, 2013

Mainz, Germany

${ }^{*}$ Speaker. 


\section{Landscape of heavy baryon spectroscopy}

Experimental observations of charmed and bottom baryons date back to the mid 1970s with the discovery of the $\Lambda_{c}^{+}$, and to the early 1990s with the discovery of the $\Lambda_{b}$. Since that time, the experimental spectrum has filled out with many precise measurements of the ground state energies of singly charmed and bottom baryons. In the past 10 years, results from BaBar and Belle, as well as the D0 and CDF experiments at Fermilab have provided more precise measurements of heavy flavor ground and excited states as well as a first glimpse of several new states, most notably the controversial $\Omega_{b}$ at CDF [1] and D0 [2] and the $\Xi_{c c}$ at SELEX [3][4]. Although the former controversy has been resolved by a more recent measurement at LHCb [5], the latter observation has still not received experimental verification to date, and is only marginally in agreement with most current theoretical results. It is controversies like these, as well as the prospect of observations of new states that make this an exciting time for lattice QCD. The opportunities for heavy flavour spectroscopy from the lattice are threefold: 1) Lattice results can help to resolve controversies like the ones mentioned above (providing a model-independent point of comparison for current experimental measurements), 2) Lattice results can help to provide predictions for as yet unobserved states and 3) Spectroscopy calculations can help to pin down values for the low energy constants (LEC's) of chiral perturbation theory (a job that lattice calculations are uniquely suited for as quark masses can be varied), helping to aid in the understanding of hadronic physics at low energies. The predictive power of lattice QCD is especially exciting in the context of the upcoming increase in operating energy at the LHC scheduled for 2014.

The interest in heavy baryon spectroscopy within the lattice community is reflected by the array of calculations of the spectra of singly, doubly, and triply heavy baryons dating back to 2002 with Ref. [6] and more recently with [7],[8] and [9]. The interest in heavy hadron spectroscopy has not been limited to the lattice community, and there has been significant recent progress in theoretical calculations of the charmed and bottom baryon spectra as well using various models [10][11][12][13]. Included among these calculations are several determinations of the doubly heavy baryons which contain charm and bottom quarks. With the exception of the early quenched heavy hadron study [6], this subsector of doubly heavy states remains largely unexplored in lattice calculations, and there is no existing experimental data on charmed bottom baryons. This is a void that the present work will seek to fill, while at the same time reinforcing the existing lattice calculations of the charmed and bottom baryon spectra with a calculation using a single action for the light quark sector.

\section{Lattice actions and parameters}

In the present work, we utilize ensembles of gauge field configurations generated by the RBC/UKQCD collaboration [14]. We work at one lattice volume, with spatial extent $L \approx 2.7$ $\mathrm{fm}$, and two lattice spacings: a fine lattice spacing with $a \approx 0.0849 \mathrm{fm}$, and a coarse lattice spacing with $a \approx 0.1119 \mathrm{fm}$. These ensembles were generated using the Iwasaki gauge action and include $2+1$ flavors of dynamical light quarks implemented with a domain-wall action. Propagators for the light up and down quarks were generated on these ensembles at both the unitary point (where the sea quark mass is equal to the valence quark mass) as well as at several other lighter masses. 
Strange quark propagators at an additional strange quark mass were computed on the coarse lattice at the light quark unitary point, allowing for an interpolation to the physical strange quark mass. The current calculation therefore includes sea-sea pion masses in the range $m_{\pi}^{s s} \approx 295 \mathrm{MeV}-352$ $\mathrm{MeV}$, while the valence-valence pion masses range from $m_{\pi}^{s s} \approx 227 \mathrm{MeV}-352 \mathrm{MeV}$. The kaon mass range is: $m_{K}^{v v} \approx 523 \mathrm{MeV}-586 \mathrm{MeV}$. This partial quenching in both the light (up and down) and strange sectors, requires special care to be taken in ensuring that chiral extrapolations are correctly performed.

For the bottom quarks, we use improved lattice NRQCD [15] accurate through order $v^{4}$ and $\Lambda_{\mathrm{QCD}}^{2} / m_{b}^{2}$, with the value for the coefficient $c_{4}$ computed specifically for the Iwasaki gauge action by Tom Hammant. As the mass of the charm quark is too light to call for a non-relativistic treatment on the current lattices, we use instead a relativistic heavy quark action in the Fermilab interpretation [16]. The action we use is of the same form as the one in Ref. [17]. To tune this action for the charm quark, the bare action parameters $v$ and $m_{0}$ were simultaneously tuned to match the spin-averaged charmonium mass and enforce the dispersion relationship, both of which are given by:

$$
M_{S A}=\frac{1}{4} M_{\eta_{c}}+\frac{3}{4} M_{J / \psi}, \quad c^{2}=\frac{E^{2}\left(\boldsymbol{p}^{2}\right)-M_{0}^{2}}{\boldsymbol{p}^{2}} .
$$

The additional bare parameters in the relativistic heavy quark action, $c_{E}$ and $c_{B}$ were set to their tree level values from lattice perturbation theory.

\section{Extracting masses}

Starting from three quark fields $q, q^{\prime}, q^{\prime \prime}$, we construct two basic types of baryon operators, given by

$$
\begin{aligned}
O_{5}\left[q, q^{\prime}, q^{\prime \prime}\right]_{\alpha} & =\varepsilon_{a b c}\left(C \gamma_{5}\right)_{\beta \gamma} q_{\beta}^{a} q_{\gamma}^{\prime b}\left(P_{+} q^{\prime \prime}\right)_{\alpha}^{c}, \\
O_{j}\left[q, q^{\prime}, q^{\prime \prime}\right]_{\alpha} & =\varepsilon_{a b c}\left(C \gamma_{j}\right)_{\beta \gamma} q_{\beta}^{a} q_{\gamma}^{\prime b}\left(P_{+} q^{\prime \prime}\right)_{\alpha}^{c},
\end{aligned}
$$

where $a, b, c$ are color indices, $\alpha, \beta, \gamma$ are spinor indices, $C$ is the charge conjugation matrix, and $P_{+}$is the positive-parity projector

$$
P_{+}=\frac{1}{2}\left(1+\gamma_{0}\right)
$$

We also compute the energies of heavy pseudoscalar and vector mesons, using two-point functions of the operators

$$
\begin{aligned}
& O_{5}^{(M)}\left[\bar{q}, q^{\prime}\right]=\bar{q} \gamma_{5} q^{\prime}, \\
& O_{j}^{(M)}\left[\bar{q}, q^{\prime}\right]=\bar{q} \gamma_{j} q^{\prime} .
\end{aligned}
$$

It turns out to be useful to form certain linear combinations of baryon and meson energies, in which the quark-mass dependence and some of the other systematic uncertainties are expected to be reduced. For hadrons containing $b$ quarks, such linear combinations can also be used to cancel the NRQCD energy shift, while for hadrons containing $c$ quarks, this can help to eliminate effects introduced by tuning the relativistic heavy quark action for the charm sector. These linear combinations are taken as follows: 


$$
M_{O}=M_{O}^{l a t t}-\frac{n_{c}}{2} M_{S A_{c}}^{l a t}-\frac{n_{b}}{2} M_{S A_{b}}^{l a t t}+\frac{n_{c}}{2} M_{S A_{c}}^{P D G}+\frac{n_{b}}{2} M_{S A_{b}}^{P D G} .
$$

Here, $n_{c, b}$ is the number of charm or bottom quarks in the baryon, and $M_{S A_{c, b}}$ is the spin averaged charmonium or bottomonium mass.

We construct an operator basis using various levels of Gaussian smearing at the source and sink. We are reusing previously computed domain wall light and strange propagators, therefore we do not have the freedom to change the source smearing on these quarks. For the heavy quarks, we establish a hierarchy of smearing by reducing the level of smearing of the charm quarks in relation to the light quarks, and reducing the level of smearing of the bottom quarks in relation to that of the charm. The reasoning behind this is that the heavier fermions are expected to have a more spatially localized wavefunction. We apply smearing to either all or none of the heavy quarks, leading to $2 \mathrm{x}$ 2 correlation function matrices for the triply heavy interpolating operators, and a $2 \times 4$ correlation function matrices for the doubly and triply heavy interpolating operators. Simultaneous fits of the resulting correlation function matrices were then carried out, allowing for variable fitting ranges for the individual correlation functions. This allows for an optimal fit range to be chosen for correlation functions with varying levels of excited state contamination. This process of choosing the optimal fit ranges was carried out by using initial guesses of the best fit ranges, and then stochastically minimizing the total $\chi^{2} /$ d.o.f. as a function of the fit ranges. An example of the effects of this minimization procedure can be seen in Figure. 1
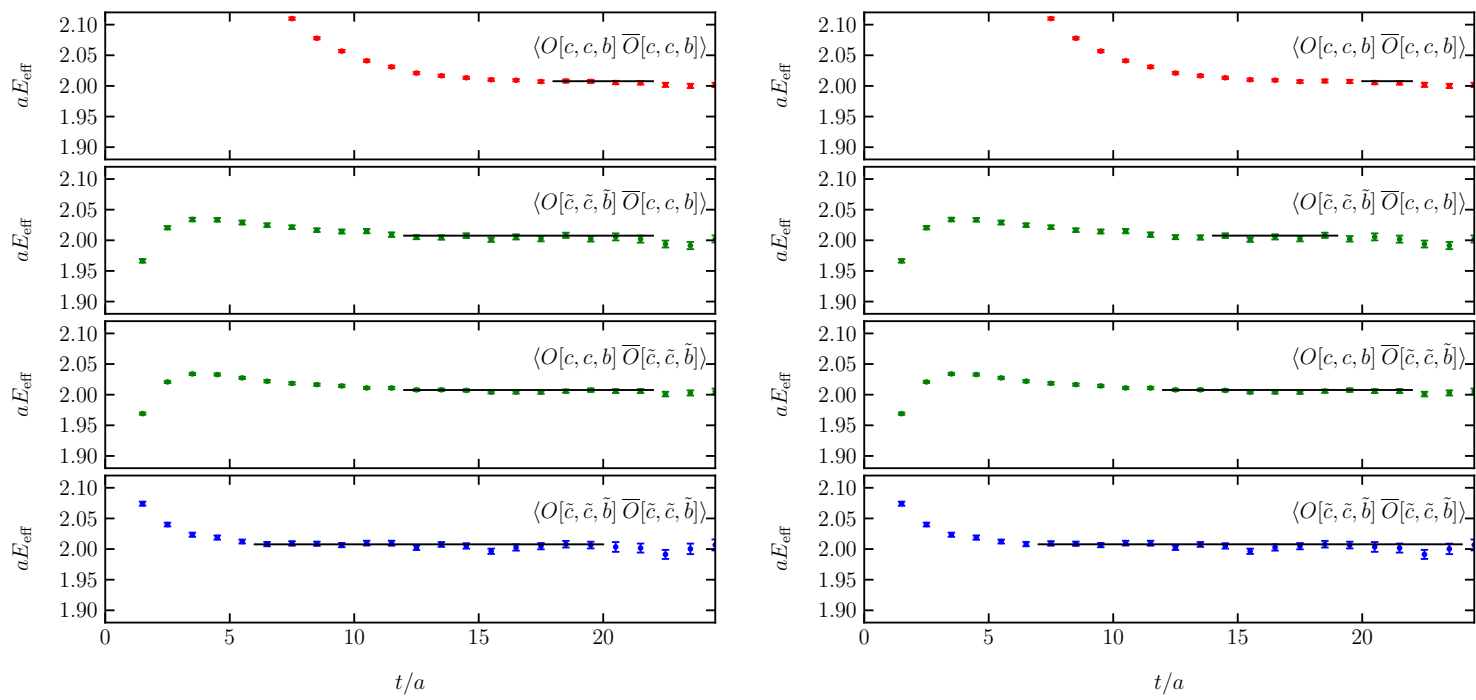

Figure 1: An illustration for the effects of the stochastic fitting range determination for the state $\Omega_{c c b}$. Here, the left hand plot corresponds to the initial guesses for fitting ranges, while the right hand plot corresponds to the optimized fitting ranges.

\section{Chiral/Continuum extrapolations}

As this spectroscopy calculation has been performed using several unphysical pion masses, in order to obtain a final result that is comparable to experimental results (as well as provide useful 
predictions for baryons that have yet to be observed) we must perform an extrapolation of our results to the physical pion mass. One complicating factor stems from the fact that the calculation was performed using partial quenching in the light quark sector in order to extend the range of pion masses beyond those offered by the set of gauge field configurations used. To perform the extrapolations, we use partially quenched heavy hadron chiral perturbation theory (PQ HH $\chi \mathrm{PT}$ ) as a guide. PQ HH $\chi$ PT constructs the chiral Lagrangian to include heavy quark symmetries in the infinite mass limit. Expressions have been derived for the sea and valence mass dependence of singly [18] and doubly heavy [19] baryon masses, the latter relying on quark-diquark symmetry to relate the properties of singly heavy mesons to doubly heavy baryons. As the chiral expansion for $\mathrm{SU}(3)$ is thought to have poor convergence properties, we perform our extrapolations using SU(4|2) HH PQ $\chi$ PT. For singly heavy baryons, we extend existing expressions [18] for the baryon masses to include $\mathscr{O}\left(1 / m_{Q}\right)$ corrections, while for the doubly heavy sector, we use the expressions presented in Ref. [19]. To include finite volume effects that appear from performing the calculation in a finite sized box, we include corrections to the chiral functions taken from Ref. [20]

In addition to the light quark mass dependence, the dependence on the finite lattice spacing must be accounted for. In order to accomplish this, we parametrize the lattice spacing dependence by adding a term of the form $c_{a} a^{2}$ to each of the chiral extrapolations. We then set $c_{a}$ to zero to obtain the results in the continuum limit.

In order to stabilize the fits, the axial couplings $g_{1}, g_{2}, g_{3}$ (included in the chiral loop terms) are constrained using Gaussian priors to be near their values as calculated for the infinite mass limit in Ref. [21]. The Gaussian width used in the constraint was taken to be the statistical uncertainty for the $g_{i}$ from Ref. [21] with an additional increase in the the width for the charmed baryons versus bottom baryons.

An example of the chiral and continuum extrapolations is presented in Figure 2.

\section{Results and future outlook}

Results for these extrapolations are presented in Tables 1 and 2. The uncertainties presented in these tables are statistical only. Systematic uncertainties are currently being explored, in both the mass extractions and chiral extrapolations. The limited mass range of $m_{\pi}^{s s}$ may be inadequate in constraining the chiral extrapolations, therefore we are currently working to supplement the data sets with an additional sea pion mass.

\begin{tabular}{|c||c|c||c||c|c|}
\hline Baryon & Lattice $(\mathrm{GeV})$ & Expt. $(\mathrm{GeV})$ & Baryon & Lattice $(\mathrm{GeV})$ & Expt. $(\mathrm{GeV})$ \\
\hline \hline$\Lambda_{c}$ & $2.137(74)$ & 2.286 & $\Lambda_{b}$ & $5.456(114)$ & 5.619 \\
$\Sigma_{c}$ & $2.444(81)$ & 2.454 & $\Sigma_{b}$ & $5.781(96)$ & 5.811 \\
$\Sigma_{c}^{*}$ & $2.518(82)$ & 2.518 & $\Sigma_{b}^{*}$ & $5.802(97)$ & 5.832 \\
$\Xi_{c}$ & $2.372(58)$ & 2.467 & $\Xi_{b}$ & $5.760(80)$ & 5.791 \\
$\Xi_{c}^{\prime}$ & $2.526(62)$ & 2.575 & $\Xi_{b}^{\prime}$ & $5.947(81)$ & - \\
$\Xi_{c}^{*}$ & $2.600(62)$ & 2.645 & $\Xi_{b}^{*}$ & $5.971(81)$ & - \\
$\Omega_{c}$ & $2.615(67)$ & 2.685 & $\Omega_{b}$ & $6.008(80)$ & 6.071 \\
$\Omega_{c}^{*}$ & $2.690(67)$ & 2.765 & $\Omega_{b}^{*}$ & $6.036(80)$ & - \\
\hline
\end{tabular}

Table 1: Chiral and continuum extrapolated results for all singly heavy states considered in the present work. 

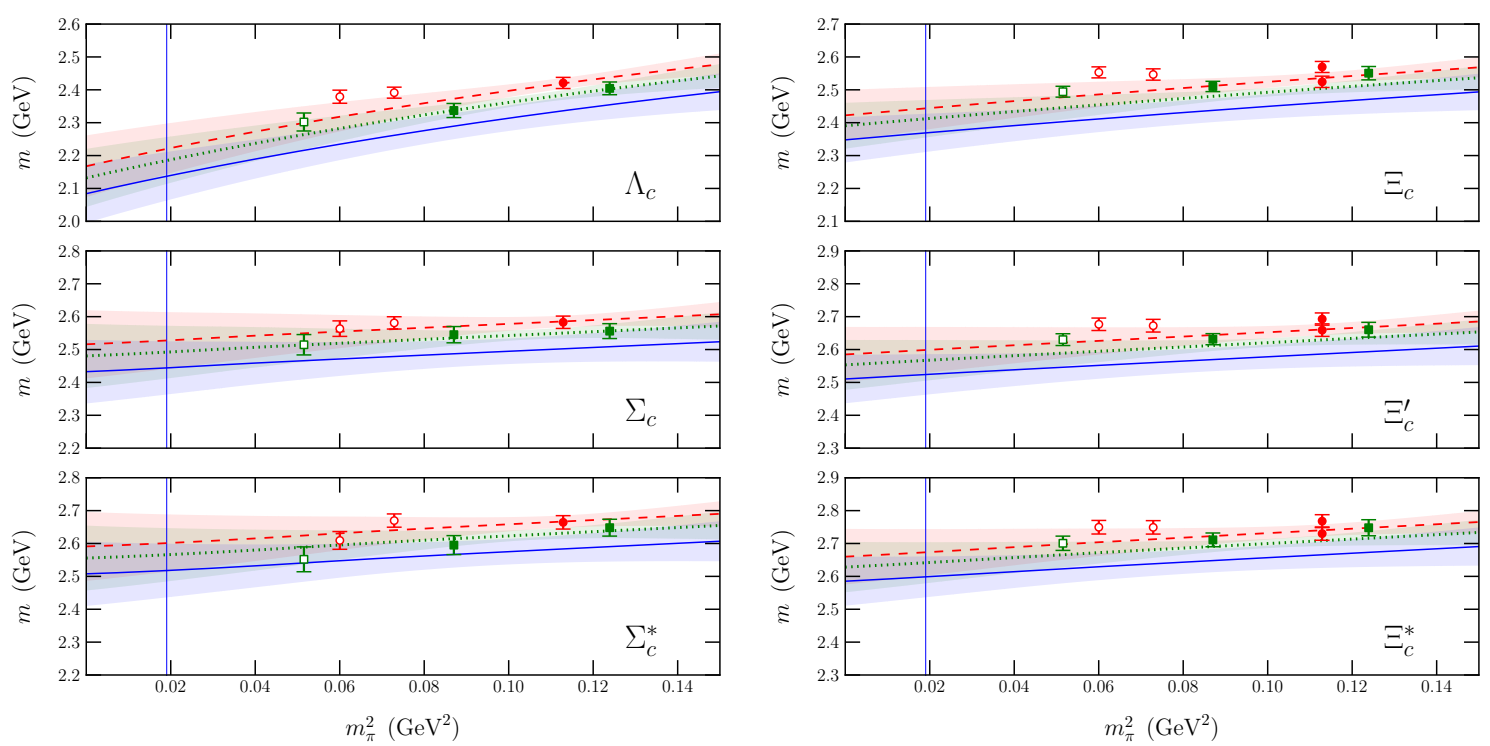

Figure 2: Example chiral and continuum extrapolations for the singly charmed states. Here, the open symbols represent partially quenched data while the filled symbols represent unitary points. The dashed line represents the coarse lattice spacing, the dotted line the fine lattice spacing and the solid line represents the continuum limit extrapolation. Both extrapolations are presented with the finite volume effects removed.

\begin{tabular}{|c||c||c||c||c||c|}
\hline Baryon & Lattice $(\mathrm{GeV})$ & Baryon & Lattice $(\mathrm{GeV})$ & Baryon & Lattice $(\mathrm{GeV})$ \\
\hline \hline$\Xi_{c c}$ & $3.558(39)$ & $\Xi_{c b}$ & $6.877(52)$ & $\Xi_{b b}$ & $10.185(53)$ \\
$\Xi_{c c}^{*}$ & $3.627(54)$ & $\Xi_{c b}^{*}$ & $6.915(62)$ & $\Xi_{b b}^{*}$ & $10.191(56)$ \\
$\Omega_{c c}$ & $3.689(38)$ & $\Omega_{c b}$ & $6.973(48)$ & $\Omega_{b b}$ & $10.250(51)$ \\
$\Omega_{c c}^{*}$ & $3.773(38)$ & $\Omega_{c b}^{*}$ & $7.040(48)$ & $\Omega_{b b}^{*}$ & $10.283(51)$ \\
$\Omega_{c c c}$ & $4.794(9)$ & $\Omega_{c c b}$ & $7.989(11)$ & $\Omega_{c c b}^{*}$ & $8.012(12)$ \\
$\Omega_{c b b}$ & $11.177(9)$ & $\Omega_{c b b}^{*}$ & $11.206(11)$ & $\Omega_{b b b}$ & $14.370(10)$ \\
\hline
\end{tabular}

Table 2: Chiral and continuum extrapolated results for all doubly and triply heavy states considered in the present work. 


\section{References}

[1] T. Aaltonen et al. Phys.Rev., vol. D80, p. 072003, 2009.

[2] V. Abazov et al. Phys.Rev.Lett., vol. 101, p. 232002, 2008.

[3] M. Mattson et al. Phys.Rev.Lett., vol. 89, p. 112001, 2002.

[4] A. Ocherashvili et al. Phys.Lett., vol. B628, pp. 18-24, 2005.

[5] D. Milanes EPJ Web Conf., vol. 28, p. 04010, 2012.

[6] N. Mathur, R. Lewis, and R. Woloshyn Phys.Rev., vol. D66, p. 014502, 2002.

[7] R. A. Briceno, H.-W. Lin, and D. R. Bolton Phys.Rev., vol. D86, p. 094504, 2012.

[8] C. Alexandrou, J. Carbonell, D. Christaras, V. Drach, M. Gravina, et al. Phys.Rev., vol. D86, p. $114501,2012$.

[9] Y. Namekawa PoS, vol. LATTICE2012, p. 139, 2012.

[10] W. Roberts and M. Pervin Int.J.Mod.Phys., vol. A23, pp. 2817-2860, 2008.

[11] A. Martynenko Phys.Lett., vol. B663, pp. 317-321, 2008.

[12] D. Ebert, R. Faustov, V. Galkin, and A. Martynenko Phys.Rev., vol. D66, p. 014008, 2002.

[13] R. Roncaglia, D. Lichtenberg, and E. Predazzi Phys.Rev., vol. D52, pp. 1722-1725, 1995.

[14] Y. Aoki et al. Phys.Rev., vol. D83, p. 074508, 2011.

[15] G. P. Lepage, L. Magnea, C. Nakhleh, U. Magnea, and K. Hornbostel Phys.Rev., vol. D46, pp. 4052-4067, 1992.

[16] A. X. El-Khadra, A. S. Kronfeld, and P. B. Mackenzie Phys.Rev., vol. D55, pp. 3933-3957, 1997.

[17] L. Liu, H.-W. Lin, K. Orginos, and A. Walker-Loud Phys.Rev., vol. D81, p. 094505, 2010.

[18] B. C. Tiburzi Phys.Rev., vol. D71, p. 034501, 2005.

[19] T. Mehen and B. C. Tiburzi Phys.Rev., vol. D74, p. 054505, 2006.

[20] W. Detmold, C.-J. D. Lin, and S. Meinel Phys.Rev., vol. D84, p. 094502, 2011.

[21] W. Detmold, C.-J. D. Lin, and S. Meinel Phys.Rev.Lett., vol. 108, p. 172003, 2012. 